\title{
Theoretical mechanistic study on the radical-molecule reaction of Ketenyl with nitrogen dioxide
}

\author{
Jia-xu Zhang, Ze-sheng Li $^{*}$, Jing-yao Liu, Chia-Chung Sun \\ Institute of Theoretical Chemistry, State Key Laboratory of Theoretical and \\ Computational Chemistry, Jilin University, Changchun 130023, P. R. China
}

Table S1. The harmonic vibrational frequencies $\left(\mathrm{cm}^{-1}\right)$ of reactants, some important

products, isomers, and transition states at the B3LYP/6-311G(d,p) level. Page S2

Figure S1. The B3LYP/6-311G(d,p) optimized geometries of unfavorable species for $\mathrm{CH}_{2} \mathrm{OH}+\mathrm{NO}_{2}$ reaction. Bond distances are in angstroms and angles are in degrees.

Page S3

${ }^{*}$ Corresponding author. Fax:+86-431-8945942. E-mail address: Zeshengli@ mail.jlu.edu.cn 
Table S1. The harmonic vibrational frequencies $\left(\mathrm{cm}^{-1}\right)$ of reactants, some important products, isomers, and transition states at the B3LYP/6-311G(d,p) level.

\begin{tabular}{|c|c|c|}
\hline Species & Frequencies & $\operatorname{Expt}^{\mathrm{a}}$ \\
\hline $\mathrm{CH}_{2} \mathrm{OH}$ & $\begin{array}{l}439,588,1061,1207,1367,1488, \\
3112,3256,3840\end{array}$ & $\begin{array}{l}420,-, 1056,1183,1357,1465, \\
-, 3650\end{array}$ \\
\hline $\mathrm{NO}_{2}$ & $767,1399,1707$ & $750,1318,1618$ \\
\hline $\mathrm{CH}_{2} \mathrm{O}$ & $1202,1270,1539,1827,2869,2919$ & $1167,1249,1500,1746,2783,2843$ \\
\hline HONO-trans & $591,619,834,1298,1793,3776$ & \\
\hline HONO-cis & $638,718,893,1338,1720,3585$ & \\
\hline $\mathrm{HNO}_{2}$ & $800,1068,1408,1543,1678,3146$ & \\
\hline $\mathrm{HOCHO}$ & $\begin{array}{l}633,702,1058,1133,1311,1411, \\
1836,3044,3737\end{array}$ & $\begin{array}{l}625,638,1033,1105,1229,1387, \\
1770,2943,3570\end{array}$ \\
\hline HNO & $1576,1673,2829$ & $1511,1569,2854$ \\
\hline 1 & $\begin{array}{l}112,239,376,489,577,698,887, \\
1064,1163,1234,1355,1409,1426, \\
1506,1661,3018,3156,3774\end{array}$ & \\
\hline $2^{a}$ & $\begin{array}{l}85,191,324,412,466,634,780, \\
1003,1083,1119,1290,1407,1438, \\
1503,1787,3026,3143,3817\end{array}$ & \\
\hline $2^{b}$ & $\begin{array}{l}153,275,345,490,539,681,855, \\
923,1058,1130,1284,1404,1428, \\
1500,1671,3047,3160,3821\end{array}$ & \\
\hline TS1/2 ${ }^{\mathrm{a}}$ & $\begin{array}{l}211 \mathrm{i}, 201,237,279,372,442,749, \\
785,834,1125,1196,1352,1400, \\
1484,1547,3232,3413,3766\end{array}$ & \\
\hline $\mathbf{T S 1} / \mathbf{P}_{1}$ & $\begin{array}{l}943 \mathrm{i}, 153,321,359,576,646,867, \\
880,1085,1214,1214,1315,1457, \\
1629,1681,2008,2964,3017\end{array}$ & \\
\hline $\operatorname{TS}^{\mathrm{a}} / 2^{\mathrm{b}}$ & $\begin{array}{l}252 \mathrm{i}, 108,245,373,508,528,790, \\
928,1046,1085,1275,1393,1437, \\
1500,1778,2991,3133,3826\end{array}$ & \\
\hline $\mathbf{T S 2}^{\mathrm{a}} / \mathbf{P}_{2}$ & $\begin{array}{l}883 \mathrm{i}, 149,271,308,465,528,592, \\
725,1072,1184,1261,1280,1367, \\
1454,1586,1926,2960,3768\end{array}$ & \\
\hline $\mathbf{T S 2}^{\mathrm{a}} / \mathbf{P}_{\mathbf{3}}$ & $\begin{array}{l}1107 i, 130,332,444,467,638,803, \\
831,1092,1227,1247,1306,1422, \\
1610,1680,1899,3009,3083\end{array}$ & \\
\hline $\mathbf{T S 2}^{\mathrm{b}} / \mathbf{P}_{4}$ & $\begin{array}{l}1254 \mathrm{i}, 204,277,341,454,585,820, \\
874,1209,1231,1277,1363,1391, \\
1449,1634,1795,3040,3134\end{array}$ & \\
\hline
\end{tabular}

${ }^{\mathrm{a}}$ Experimental values from ref. 37. 
Figure S1.

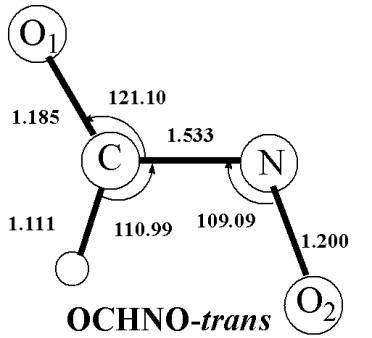

$\left(\mathrm{C}_{\mathrm{s}}\right)$

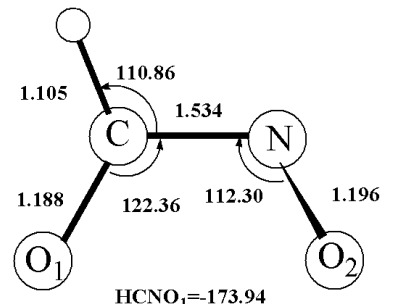

OCHNO-cis

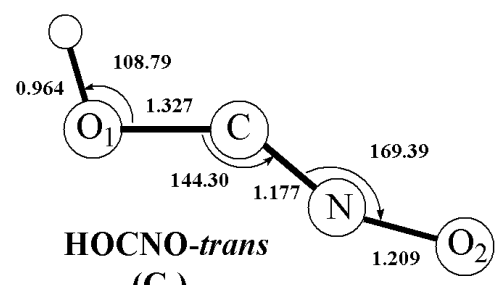

$\left(\mathrm{C}_{\mathrm{s}}\right)$

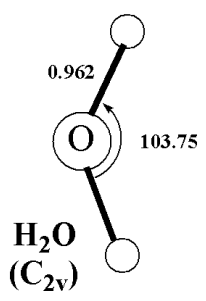

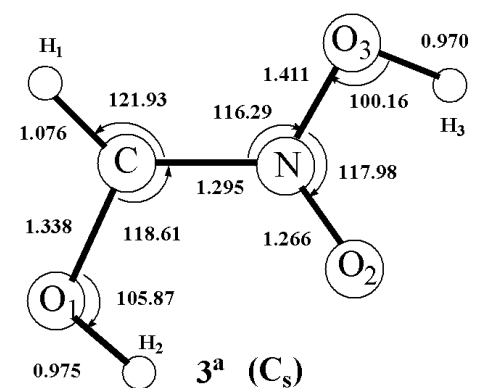
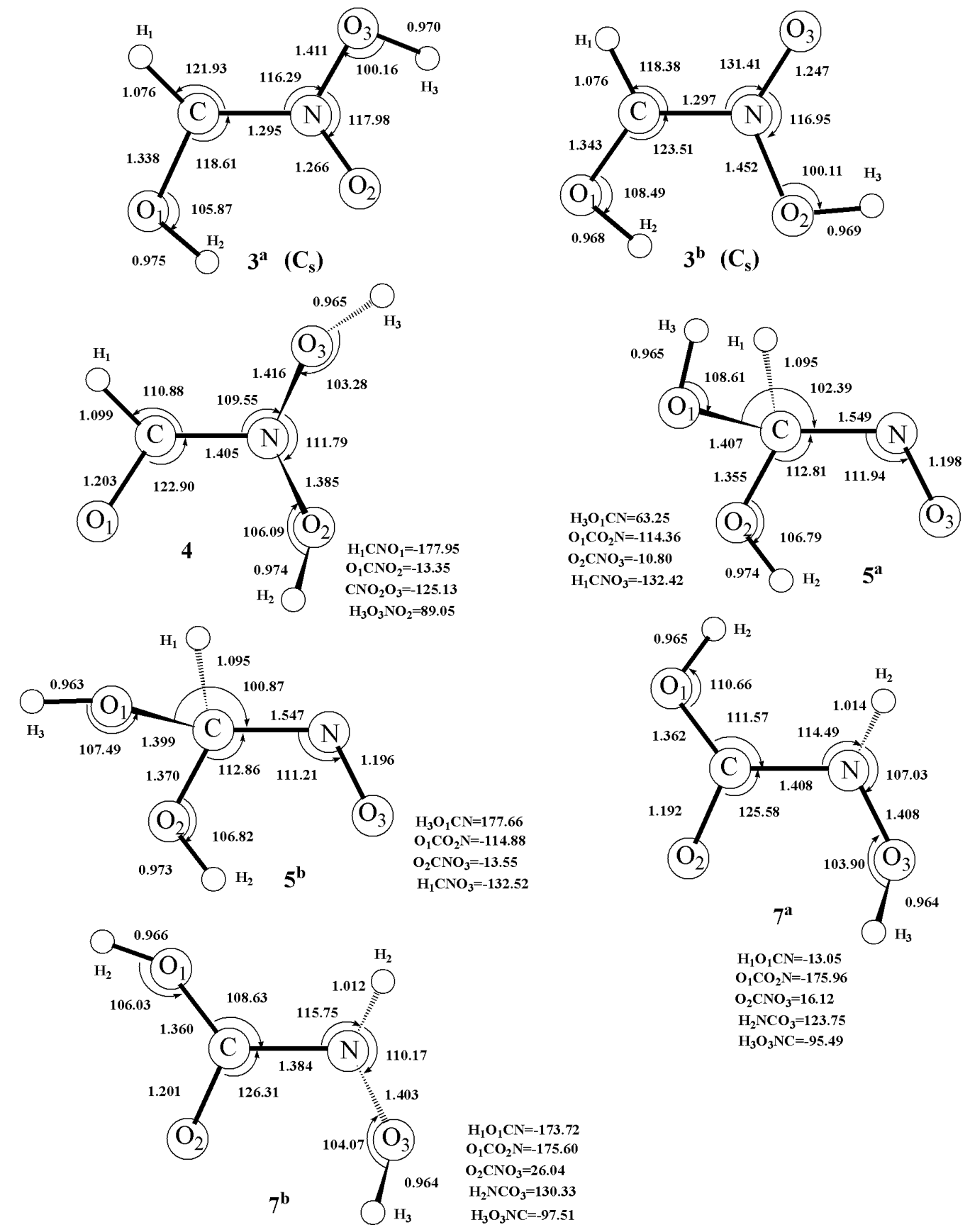

$\mathrm{H}_{1} \mathrm{O}_{1} \mathrm{CN}=-13.05$

$\mathrm{O}_{1} \mathrm{CO}_{2} \mathrm{~N}=-175.96$

$\mathrm{O}_{2} \mathrm{CNO}_{3}=16.12$

$\mathrm{H}_{2} \mathrm{NCO}_{3}=123.75$

$\mathrm{H}_{3} \mathrm{O}_{3} \mathrm{NC}=-95.49$ 